\title{
Pulse Pressure and Michigan Neuropathy Screening Instrument are Independently Associated with Asymptomatic Peripheral Arterial Disease among Type 2 Diabetes Community Residents: A Community-based Screening Program in Taiwan
}

\author{
Li-Chi Fan ${ }^{1}$, Mei-Yen Chen ${ }^{2}$, Wei-Chao Huang ${ }^{3}$, Cheng Ho ${ }^{1}$, Pao-Yin Chen ${ }^{1}$, \\ Jui-Chu Huang ${ }^{1}$, Hsu-Huei Weng ${ }^{4}$, Yun-Shing Peng ${ }^{1}$
}

Background: Peripheral arterial disease (PAD) is one of the major manifestations of systemic atherosclerosis and plays an important role in low-extremity amputation in type 2 diabetic patients. The aim of this study was to explore the prevalence and risk factors for asymptomatic PAD in type 2 diabetic community residents.

Methods: This cross-sectional study enrolled 552 type 2 diabetic adults (232 men and 320 women) without subjective symptoms of intermittent claudication. We defined the PAD group as an ankle-brachial index $(\mathrm{ABI}) \leq 0.90$, and the normal group as an ABI 0.91-1.30. Their clinical characteristics, Michigan Neuropathy Screening Instrument (MNSI) scores and blood pressure were compared.

Results: We discovered that 51 patients have asymptomatic PAD. Univariate logistic regression analysis revealed that age, history of stroke, longer duration of diabetes ( $>10$ years), unemployment or retirement, pulse pressure, systolic blood pressure, and high MNSI score ( $>2$ ) were risk factors for PAD. By multivariate logistic regression analysis, pulse pressure, high MNSI score, age, and history of stroke were independent risk factors with odds ratios (95\% confidence intervals, CI) of 1.032 (1.012-1.053), 2.359 (1.274-4.370), 1.050 (1.010-1.091), and 5.152 (1.985-13.368), respectively. Furthermore, the prevalence of PAD increased significantly with increment in the pulse pressure and MNSI.

Conclusions: In summary, the overall prevalence of asymptomatic PAD in the type 2 diabetic adults was 9.2\%. Age, history of stroke, pulse pressure and MNSI score may provide important clinical information. Primary care physicians should be aware of asymptomatic patients with high pulse pressure and MNSI scores. (Biomed J 2013;36:282-288)

Keywords: asymptomatic, diabetes mellitus, Michigan Neuropathy Screening Instrument, peripheral arterial disease, pulse pressure

From the ${ }^{1}$ Division of Endocrinology and Metabolism, Department of Internal Medicine, Chang Gung Memorial Hospital at Chiayi, Chang Gung University College of Medicine, Taoyuan, Taiwan; ${ }^{2}$ College of Nursing and the Chronic Disease and Health Promotion Center, Chang Gung University of Science and Technology, Taoyuan, Taiwan; ${ }^{3}$ Division of Plastic and Reconstructive Surgery,

Department of Surgery, Buddhist Tzu Chi General Hospital, Taipei, Taiwan; ${ }^{4}$ Department of Diagnostic Radiology, Chang Gung Memorial Hospital at Chiayi, Chang Gung University College of Medicine, Taoyuan, Taiwan

Received: Mar. 29, 2012; Accepted: Nov. 15, 2012

Correspondence to: Dr. Yun-Shing Peng, Division of Endocrinology and Metabolism, Department of Internal Medicine, Chang Gung Memorial Hospital at Chiayi. 6, W. Sec., Jiapu Rd., Puzi, Chiayi 613, Taiwan (R.O.C.). Tel: 886-5-3621000 ext. 2029; Fax: 886-5-3620311; E-mail: pengys@adm.cgmh.org.tw

DOI: $10.4103 / 2319-4170.113371$

\begin{abstract}
At a Glance Commentary
Scientific background of the subject

Peripheral arterial disease (PAD) plays a major role in low-extremity ampuin type 2 diabetic patients. Most of the American Diabetes Association recomperforming a comprehensive foot to identify risk factors predictive of ulcers and amputation.

\section{What this study adds to the field}

The prevalence of asymptomatic PAD in type 2 diabetic community residents was . It increased significantly with increPrimary care physicians should be aware of asymptomatic patients with high pulse pressure or MNSI scores.
\end{abstract}


$\mathrm{P}$ eripheral arterial disease (PAD) is one of the major manifestations of systemic atherosclerosis. ${ }^{[1]}$ In diabetes, the onset of PAD may be earlier, and may consist of more asymptomatic patients. In addition, the progression of PAD may be more rapid with poorer outcomes. ${ }^{[1-4]}$ PAD also plays a major role in low-extremity amputation in these patients..$^{[1,4,5]}$

For decades, the ankle-brachial index (ABI) has been used as a simple, rapid and effective tool for PAD screening. ${ }^{[6]} \mathrm{ABI}$ shows high sensitivity and specificity with little variability. ${ }^{[7,8]}$ The American Heart Association guidelines suggest that the ABI should be used to screen patients with symptoms of PAD, those older than 70 years of age, and those with risk factors (including diabetes, smoking, hypertension, dyslipidemia, and hyperhomocysteinemia) ${ }^{[9]}$ The guidelines also suggest that the ABI can be considered as an effective tool for cardiovascular risk assessment. ${ }^{[9]}$

Several studies have surveyed PAD in the community. ${ }^{[6]}$ These studies have mainly focused on the quality of life and ethnic differences, as well as cardiovascular outcomes. ${ }^{[10,11]}$ While many studies have documented the value of the ABI as a screening tool in the community, fewer studies have focused on performing the ABI on asymptomatic PAD in type 2 diabetic individuals. The primary goal of this study was to explore the prevalence and risk factors for asymptomatic PAD in type 2 diabetic patients.

\section{METHODS}

\section{Design}

This community-based screening program was established by Chiayi Chang Gung Medical Hospital, Chang Gung Institute of Nursing and the Chiayi County Health Bureau. The institutional ethics committee reviewed and approved the study.

From March 2009 to August 2010, we set up stations in health care centers in the 18 townships of Chiayi County. Seven hundred and sixteen diabetic adults were recruited into the screening program. Most of these patients (92\%) were retired elderly, farmers, fishermen, aquaculture workers or housewife.

We used questionnaires to assess baseline characteristics, risk factors and variables relating to diabetic foot care. Risk factors included age, gender, body mass index (BMI), history of coronary heart disease, stroke, PAD, hypertension, duration of diabetes, smoking and alcohol consumption. Since no blood was drawn for laboratory examinations during the screening process, participants were asked to bring their previous laboratory data (fasting plasma glucose, glycosylated hemoglobin and lipid profile) to the health care centers. Trained health care staff performed diabetic foot examinations, including inspection, evaluation of foot ulcers, checking ankle reflexes and measuring the vibratory perception threshold at the lateral malleolus with a tuning fork. The MNSI score was then calculated. Our trained nurses performed ankle-brachial index measurement. After completion of assessment, the doctors explained the results to the participants. Further cardiologist visits were suggested to the high-risk patients.

Because the purpose of this study was to examine the risk factors in asymptomatic adults with type 2 diabetes for PAD. Participants were excluded if they had subjective symptoms of intermittent claudication or a history of PAD, subclavian or brachiocephalic occlusive disease, proximal iliac artery disease. We also excluded those who did not: (1) complete the questionnaires (with regards to age, intermittent claudication, history of foot ulcers, history of previous amputations); (2) complete height, weight and blood pressure recording; and (3) have MNSI and ABI measurements. In total, 552 type 2 diabetic patients completed the entire screening process.

\section{Definitions and measurements}

Type 2 diabetes mellitus (DM) was defined as a first diagnosis of diabetes after the age of 30. Intermittent claudication was defined as pain, aching, or cramping of the lower limbs during movement which was relieved by rest. ${ }^{[1]}$

The nursing staff recorded the appearance of the lower limbs by inspection and calculated the MNSI score. ${ }^{[12]}$ The presence of dryness, calluses, infections, fissures or deformities was regarded as abnormal in appearance (normal $=0$, abnormal $=1)$. The presence of ulcers was recorded (absent $=0$, present $=1$ ). Ankle reflex was examined by tapping the Achilles tendon with a hammer (present $=0$, present with reinforcement $=0.5$, absent $=1$ ). Vibration perception of the toe was conducted with a $128-\mathrm{Hz}$ turning fork (present $=0$, reduced $=0.5$, absent $=1$ ) on the dorsum of the big toe. Touch-pressure sensation was tested with a $10 \mathrm{~g}$ Semmes-Weinstein monofilament by applying the monofilament perpendicular to the plantar surface (nor$\mathrm{mal}=0$, reduced $=0.5$, absent $=1$ ). The MNSI score was calculated from summation (maximal score $=10$ ) ${ }^{[12]} \mathrm{Pa}-$ tients with positive results on the MNSI score $(>2)$ were considered as having lower limbs neuropathy. ${ }^{[12,13]}$

An automated device, Cardio-Vision Model (MS-2000), was used to measure the systolic blood pressure (SBP) and diastolic blood pressure (DBP) in a supine position after a 5-minute rest. Pulse pressure was calculated as the difference between SBP and DBP on the right arm. Mean arterial pressure (MAP) was calculated as: DBP + 1/3 (SBP - DBP).

$\mathrm{ABI}$ was recorded as the ratio of ankle systolic blood pressure divided by the brachial systolic pressure. We defined the PAD group as having an $\mathrm{ABI} \leq 0.90$ and the normal group as having an ABI 0.91-1.30. ${ }^{[9]}$ Patients with an ABI of more than 1.30 were not included in the normal group due to the possibility of arterial calcification. ${ }^{[14]}$ Those who 
had no intermittent claudication and had an $\mathrm{ABI} \leq 0.9$ were defined as asymptomatic PAD. ${ }^{[1]}$

\section{Statistical analysis}

Most results were expressed as median with interquartile range (IQR) due to non-normal distribution, unless specified otherwise. Continuous variables were compared using the Mann-Whitney $U$ test and categorical data were tested with the Chi-square $\left(\chi^{2}\right)$ test or Fisher's exact test. Risk factors were assessed using univariate analysis. Variables that were statistically significant in the univariate analysis were entered into the multivariate logistic regression analysis. The presence of PAD (ABI $\leq 0.90)$ was regarded as a dependen $t$ variable and its relevant risk factors were counted for their association, using odds ratios and $95 \%$ confidence intervals. All statistical tests were two-tailed and a $p<0.05$ was considered significant. Data were analyzed using SPSS version 18.0 for Windows (SPSS Inc., Chicago, IL).

\section{RESULTS}

Seven hundred and sixteen diabetic adults participated in the screening program. We excluded 5 patients with diabetes mellitus diagnosed before the age of 30 . Twenty-four patients with a history of PAD, and 66 patients with intermittent claudication were excluded. Eight patients were also excluded due to having an $\mathrm{ABI}>1.3$, and 97 participants were not included for analysis due to the incompletion of the questionnaires. In total, 552 adults with type $2 \mathrm{DM}$ (232 men and 320 women) with asymptomatic PAD were included for further analysis.

The characteristics of the participants are listed in the Table 1. The median age was 69.5 (IQR: 62-76) years old

Table 1: Characteristics of the peripheral arterial disease group (ankle-brachial index $\leq 0.9)$ and normal group $(\mathrm{ABI}=0.91-1.3)$

\begin{tabular}{|c|c|c|c|c|}
\hline Variables & $\begin{array}{l}\text { All patients } \\
\quad(n=552)\end{array}$ & $\begin{array}{c}\mathrm{ABI} \leq 0.9 \\
(n=51)\end{array}$ & $\begin{array}{c}\mathrm{ABI}=0.91-1.3 \\
(n=501)\end{array}$ & $p$ value \\
\hline Age (years) & $69.5(62-76)$ & $76(70-80)$ & $69(62-75)$ & $<0.001$ \\
\hline Gender (Male/Female) & $232 / 320$ & $23 / 28$ & 209/292 & NS $(0.641)$ \\
\hline Body mass index $\left(\mathrm{kg} / \mathrm{m}^{2}\right)$ & $25.7(23.3-28.3)$ & $25.5(22.9-29.1)$ & $25.7(23.4-28.2)$ & NS (0.945) \\
\hline Diabetes duration (years) & $7(4-13)$ & $10(5-13)$ & $7(4-12)$ & NS $(0.060)$ \\
\hline Diabetes duration $>10$ years & $182(33 \%)$ & $25(49 \%)$ & $157(31.3 \%)$ & 0.010 \\
\hline Smoking $(\%)$ & $59(10.7 \%)$ & $5(9.8 \%)$ & $54(10.8 \%)$ & NS $(0.830)$ \\
\hline Insulin therapy (\%) & $29(5.3 \%)$ & $3(5.9 \%)$ & $26(5.2 \%)$ & NS $(0.835)$ \\
\hline $\mathrm{SBP}(\mathrm{mmHg})$ & $140.6 \pm 19.7$ & $149.6 \pm 24.6$ & $139.7 \pm 19.0$ & 0.007 \\
\hline $\mathrm{DBP}(\mathrm{mmHg})$ & $78(72-85)$ & $79(72-88)$ & $78(72-84)$ & NS (0.707) \\
\hline MAP $(\mathrm{mmHg})$ & $99.3 \pm 12.7$ & $102.9 \pm 17.4$ & $98.9 \pm 12.1$ & NS (0.116) \\
\hline Pulse pressure (mmHg) & $61(52-71)$ & $66(59-81)$ & $60(51.5-70)$ & $<0.001$ \\
\hline Cholesterol (mg/dl) & $195(169-215)$ & $196.5(176-224.75)$ & $194.5(169-214)$ & NS $(0.355)$ \\
\hline Triglyceride (mg/dl) & $116(86-160)$ & $140.5(101.7-171.3)$ & $114(83-159.5)$ & NS (0.092) \\
\hline HDL cholesterol (mg/dl) & $51.4 \pm 14.8$ & $45.2 \pm 7.5$ & $52.0 \pm 15.2$ & 0.001 \\
\hline LDL cholesterol (mg/dl) & $115.1 \pm 33.5$ & $120.9 \pm 30.4$ & $114.5 \pm 33.8$ & NS $(0.276)$ \\
\hline FPG $(\mathrm{mg} / \mathrm{dl})$ & $134(113-168.75)$ & $144.5(115.7-168.5)$ & $133(113-168.7)$ & NS (0.426) \\
\hline $\mathrm{HbA} 1 \mathrm{C}$ & $7.6(6.8-8.7)$ & $7.9(7.2-8.6)$ & $7.5(6.8-8.7)$ & NS (0.119) \\
\hline $\mathrm{ABI}$ & $1.06(0.98-1.11)$ & $0.81(0.75-0.88)$ & $1.07(1.02-1.11)$ & $<0.001$ \\
\hline MNSI score & $2(0-3)$ & $3(1-4)$ & $2(0-3)$ & $<0.001$ \\
\hline High MNSI score (>2) $(\%)$ & $182(33 \%)$ & $28(54.9 \%)$ & $154(30.7 \%)$ & $<0.001$ \\
\hline Abnormal appearance & $265(48 \%)$ & $28(54.9 \%)$ & $237(47.3 \%)$ & NS $(0.301)$ \\
\hline Foot ulcer & $5(0.9 \%)$ & $1(2 \%)$ & $4(0.8 \%)$ & NS $(0.385)$ \\
\hline Abnormal tendon reflex & $233(42.2 \%)$ & $30(58.8 \%)$ & $203(40.5 \%)$ & 0.012 \\
\hline Abnormal vibration & $100(18.1 \%)$ & $21(41.2 \%)$ & $79(15.8 \%)$ & $<0.001$ \\
\hline Abnormal touch-pressure & $108(19.6 \%)$ & $17(33.3 \%)$ & $91(18.2 \%)$ & 0.009 \\
\hline History of stroke (\%) & $23(4.2 \%)$ & $8(15.7 \%)$ & $15(3 \%)$ & $<0.001$ \\
\hline History of IHD (\%) & $81(14.7 \%)$ & $12(23.5 \%)$ & $69(13.8 \%)$ & NS $(0.061)$ \\
\hline History of hypertension (\%) & $294(53.3 \%)$ & $33(64.7 \%)$ & $261(52.1 \%)$ & NS (0.086) \\
\hline Blackfoot-townships (\%) & $73(13.2 \%)$ & $8(15.7 \%)$ & $65(13 \%)$ & NS (0.586) \\
\hline Well-educated (\%) & $123(22.3 \%)$ & $8(15.7 \%)$ & $115(23 \%)$ & NS $(0.235)$ \\
\hline Unemployment or retirement & $353(63.9 \%)$ & $42(82.4 \%)$ & $311(62.1 \%)$ & 0.004 \\
\hline
\end{tabular}

Abbreviations: NS: Not significant; M: Male; F: Female; MNSI: Michigan neuropathy screening instrument; ABI: Ankle-brachial index; SBP: Systolic blood pressure; DBP: Diastolic blood pressure; MAP: Mean arterial pressure; HDL: High-density lipoprotein; LDL: Low-density lipoprotein;

FPG: Fasting plasm glucose; HbA1C: Glycosylated hemoglobin; IHD: Ischemic heart disease; Blackfoot-associated townships: Budai and Yizhu townships

Biomed J Vol. 36 No. 6

November - December 2013 
with a median duration of diabetes of 7 (IQR: 4-13) years. Seventy-eight percent were under-educated (below elementary school or illiterate), $63.9 \%$ were unemployed or retired and the rest were mostly farmers, workers or fishermen.

An $\mathrm{ABI} \leq 0.9$ was noted in $9.2 \%$ participants (51/552: 23 men and 28 women). The prevalence of PAD was highest $(13.5 \%)$ in patients $70-89$ years old. The main differences in characteristics between the PAD group and the normal group were older age, longer duration of diabetes ( $>10$ years), alcohol consumption, history of stroke and unemployment or retirement. No statistical significance was found with respect to gender, history of ischemic heart disease or hypertension, betel nut chewing, education level, insulin therapy, BMI or smoking in our study. Some of the participants lived in the townships (Yizhu and Budai) associated with black foot disease (an endemic PAD in Taiwan due to arsenic exposure), however the results revealed no difference in the residency between the PAD and the normal groups.

Detailed comparison on each item of MNSI score was listed in Table 1. The PAD group had significant higher percentage of abnormal tendon reflexes, vibration perception and touch-pressure sensation. But the differences on appearance and current foot ulcer were not significant. The percentage of high MNSI scores ( $>2)$ was significantly higher in the PAD group compared to the normal group $(54.9 \%$ versus $30.7 \%, p<0.001)$, as were $\mathrm{SBP}(149.6 \pm 24.6 \mathrm{mmHg}$ versus $139.7 \pm 19.0 \mathrm{mmHg}$, $p=0.007)$ and pulse pressure $(66 \mathrm{mmHg}$ and IQR: 59-81 versus $60 \mathrm{mmHg}$ and IQR: $51.5-70$ ). In addition, the PAD group had a higher percentage of unemployed or retired individuals compared to the normal group ( $82.4 \%$ vs. $62.1 \%, p=0.004)$.

Multivariate logistic regression analysis was then performed using the positive risk factors in the univariate logistic regression. Independent risk factors for PAD were age (OR: $1.050,95 \%$ CI: 1.010-1.091, $p=0.013$ ), past history of stroke (OR: $5.152,95 \%$ CI: $1.985-13.368, p=0.001$ ), a high MNSI score (OR: 2.359, 95\% CI: 1.274-4.370, $p=0.001)$ and pulse pressure (OR: $1.032,95 \%$ CI: $1.012-$ $1.053, p=0.002$ ) [Table 2].

The prevalence of PAD increased significantly $(p=0.001)$ with the increments of pulse pressure by Chi-square test for trend [Figure 1]. The prevalence of PAD was $4.6 \%, 6.9 \%, 9.7 \%$ and $15.4 \%$ in each quartile of pulse pressure (cutoff points: $52 \mathrm{mmHg}, 61 \mathrm{mmHg}$ and $71 \mathrm{mmHg}$ ), respectively. Furthermore, the prevalence of PAD also increased significantly in patients with higher MNSI scores. The prevalence of PAD was $4.9 \%, 7.3 \%, 10.9 \%$ and $21 \%$, for MNSI score of $0,0.5-2$, 2.5-3, and $>3$, respectively (Chi-square test for trend, $p<0.001$ ) [Figure 2].

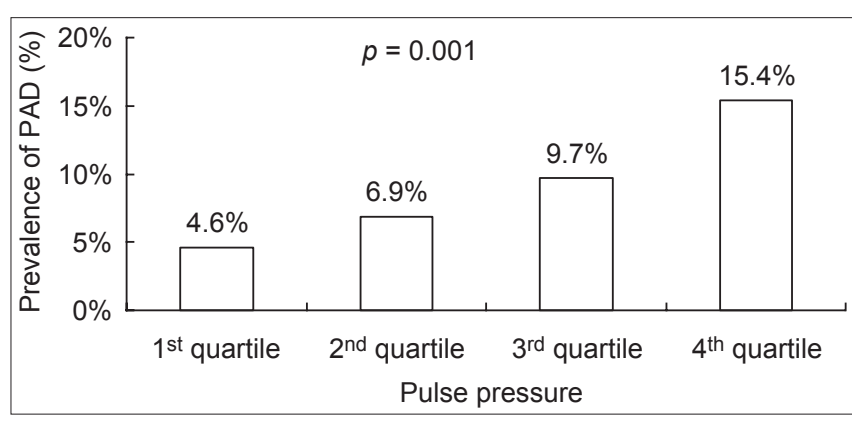

Figure 1: The prevalence of peripheral arterial disease by each quartile of pulse pressure.

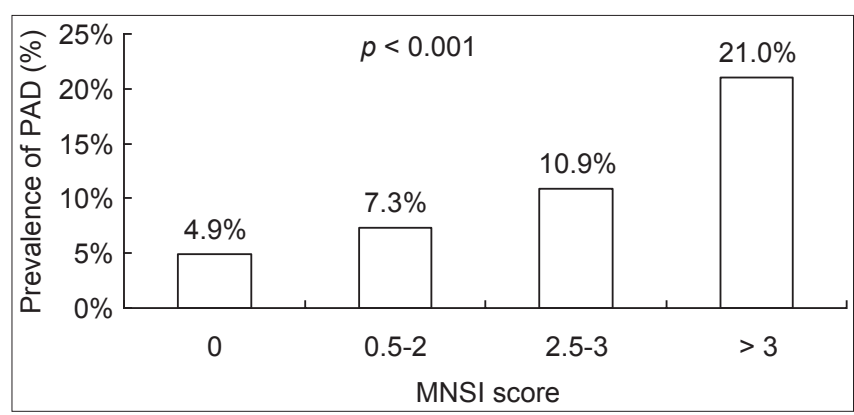

Figure 2: The prevalence of peripheral arterial disease increased significantly with the increments of michigan neuropathy screening instrument score. The chi-square test for trend was used.

Table 2: Multivariate logistic regression analysis for the variables associated with peripheral arterial disease

\begin{tabular}{lcc}
\hline Parameters & Odds ratio $(95 \% \mathrm{CI})$ & $p$ value \\
\hline Pulse pressure $(\mathrm{mmHg})$ & $1.032(1.012-1.053)$ & 0.002 \\
Age (years) & $1.050(1.010-1.091)$ & 0.013 \\
High MNSI score $(>2)$ & $2.359(1.274-4.370)$ & 0.006 \\
History of stroke & $5.152(1.985-13.368)$ & 0.001
\end{tabular}

Abbreviations: NS: Not significant; M: Male; F: Female;

MNSI: Michigan neuropathy screening instrument; ABI: Ankle-brachial index; SBP: Systolic blood pressure; DBP: Diastolic blood pressure;

MAP: Mean arterial pressure

After completion of assessment, the doctors explained the results to all participants. Further cardiologist visits were suggested to the high-risk patients. Twenty-six of the 51 patients $(51 \%)$ with an $\mathrm{ABI} \leq 0.9$ were referred to cardiology outpatients clinics of the regional teaching hospital and the peripheral arterial duplex studied were performed for confirmation. All of them received medication therapy, including anti-platelet, anti-hypertensive or lipid-lower agents. Only two patients (3.9\%) received percutaneous transluminal angioplasty (PTA) successfully. Three patients $(5.9 \%)$ refused PTA.

\section{DISCUSSION}

Most patients with PAD do not have typical intermittent claudication, ${ }^{[1,2]}$ and the $\mathrm{ABI}$ is recommended as a 
non-invasive screening tool for early detection. ${ }^{[10]}$ In our study, we examined the prevalence of PAD in asymptomatic type 2 diabetic patients and found that its occurrence was significantly associated with pulse pressure, age, history of stroke and a high MNSI score in multivariate logistic regression analysis.

The prevalence of PAD in type 2 diabetic patients is largely affected by the type of screening tool, the feature of the population and the variation in different ethnic groups. While using the $\mathrm{ABI}$ as a screening tools, the prevalence has been found to be $26.3 \%$ in Germany, ${ }^{[15]} 16 \%$ in Malaysia, ${ }^{[16]}$ $12.7 \%$ in the elderly ( $>65 \mathrm{y} / \mathrm{o})$ and $4.0 \%$ in younger adults in $\operatorname{Japan}^{[17]}$ and $17.7 \%$ in a multi-country study in Asia. ${ }^{[18]}$ In Taiwan, Tseng et al., reported the prevalence of PAD to be $10.0 \%$, based on a survey in the diabetic clinic of a teaching hospital in Taipei. ${ }^{[19]}$ The prevalence in our study was slightly lower than Tseng's study, because our study was limited to asymptomatic adults in contrast to Tseng's study in the general population. In addition, patients with PAD may lack the motivation to attend the study actively due to the absence of typical symptoms. ${ }^{[1]}$

The finding that $9.2 \%$ of type 2 diabetic adults have asymptomatic PAD which representatives multiple meanings. Seeing that there is still substantial population of asymptomatic adults, an early screening tool may greatly improve the effectiveness of PAD prevention. However, even though $\mathrm{ABI}$ is a well-validated screening tool for $\mathrm{PAD}$, it remains not conducted routinely in the community. One way to compensate this shortcoming may be relied on the local health care centers to carry on more active screening services and the prevalence of $9.2 \%$ may implicate that this type of screening is worthwhile. The health care costs may also be reduced, especially in considering the burdens of late PAD and the inexpensiveness of screening tool. ${ }^{[20]}$

Several studies have found an association of high pulse pressure with large artery stiffness ${ }^{[21]}$ higher mortality (overall, cardiovascular and cerebrovascular diseases $)^{[22-25]}$ and higher risks of foot ulcers. ${ }^{[26]}$ However, most of these studies have been targeted at the general diabetic population. In our study, the level of pulse pressure contributed independently as a risk factor (OR: 1.032 for every 1 -mmHg increment; 95\% CI: 1.012-1.053, $p=0.002$ ) for asymptomatic PAD in type 2 diabetic adults. Similarly, Tseng et al., reported that an adjusted OR for PAD was 1.035 (1.012-1.058) for every $1-\mathrm{mmHg}$ increment of pulse pressure in type 2 diabetic patients. ${ }^{[27]}$ The main difference is that our study demonstrated the significance of pulse pressure in asymptomatic PAD, thereby broadening the application of its role as a predictor. In addition, a study by Halon et al., focused on the relationship between pulse pressure and subclinical disease. ${ }^{[28]}$ They found that elevated pulse pressure was an independent marker of the presence of pre-clinical coronary artery disease in an asymptomatic diabetic population, using 64-channel cardiac computed tomography analysis. Although the instruments for detection were not the same, both studies showed that pulse pressure can be an early clinical clue of asymptomatic atherosclerosis in coronary or peripheral circulation.

The MNSI score was composed of two portions: The self-administered symptom questionnaires and the clinical examination. In this study, we only used the clinical examination portion of MNSI, which was validated as an effective surrogate for diabetic polyneuropathy. ${ }^{[29]}$ In our study, 33\% (182 of 552) patients had a high MNSI score (>2) and in these patients, $15.4 \%$ (28 of 182) had asymptomatic PAD. Moreover, we found that a high MNSI score was an independent risk factor for asymptomatic PAD (OR: $2.359 ; 95 \%$ CI: 1.274 to $4.370 ; p=0.006$ ). The link between the abnormality of MNSI score and the presence of PAD cannot be over-interpreted. Because there are many potential confounding factors in this association, a cross-sectional study is not an appropriate design to determine the casual relationship. We speculated that the patient's glycemic control and the degree of oxidative stress may be confounders in the relationship of high MNSI score and PAD, however further studies are needed to clarify this.

Aging is a well-known risk factor for PAD. ${ }^{[1,9]}$ In our results, aging remained an independent risk factor in the multivariate logistic regression. We found that the prevalence of asymptomatic PAD (14.5\%) was higher in the group more than 70 years of age. This finding supports the importance of a more active screening strategy in the elderly. On the other side, the duration of diabetes showed significant correlation with PAD by univariate analysis, but the correlation was not found in the multivariate logistic regression. This may be partly explained by the underlying association between age and duration of diabetes.

A history of stroke was significantly associated with asymptomatic PAD in this study. This association has also been found in other studies. ${ }^{[30,31]}$ One recent prospective cohort study reported that asymptomatic PAD was independently associated with recurrent vascular events and stroke with a significant hazard ratio (HR: $5.2 ; 95 \%$ CI: 1.5 to 17.6; $p=0.008$ ) in patients with stroke or transient ischemic attack. ${ }^{[31]}$ Another hospital-based study examined at the relationship between subclinical PAD and acute stroke or transient ischemic attack (TIA) ${ }^{[32]}$ The results showed that only $6.2 \%$ of these patients had a history of PAD but $44.9 \%$ actually had an $\mathrm{ABI} \leq 0.9$. These findings suggested that in patients with a history of stroke, acute stroke or TIA, ankle-brachial examinations may discover undiagnosed asymptomatic PAD. 
Blackfoot disease was once an endemic peripheral arterial disease in the southwest of Taiwan. The prevalence of blackfoot disease was estimated to be 6.51 to 18.85 per 1,000 populations in different villages, but the incidence had declined dramatically after changing the water source from artesian wells to tap water. ${ }^{[33]}$ As our study included this area (Budai Townships and Yizhu Township, with 73 participants) of endemic blackfoot disease in our study, we took the participants' residency into analysis. However, no differences were found between these and other townships. This is probably because the influence of blackfoot disease on the occurrence of PAD has decreased steadily over the decades since changing the water supply. Another reason may be that the scale of our screening program in these areas was not large enough to detect the influence of blackfoot disease on PAD.

We did not find a significant association between PAD and a history of ischemic heart disease and smoking in the univariate and multivariate logistic regression analysis. This may be due to under-reporting of the patients while completing the questionnaires.

There are several limitations to this study. First, selection bias could not be avoided because these participants were recruited from the local health care bureaus' registry. In addition, the patients who actively participated in the screening program may have had less severe disease and a greater intention to join a screening program. Second, pulse pressure measurements can be affected by exercise, excitement and medications, and these factors were not taken into consideration. Third, because our study subjects were recruited from the community and blood sampling was not included as a part of the screening program, several important laboratory variables were not as easy accessible as in a hospital-based setting.

\section{CONCLUSIONS}

In summary, PAD was found in $9.2 \%$ of type 2 diabetic patients without intermittent claudication or a history of PAD. Age, history of stroke, pulse pressure and MNSI score may provide important clinical information. Primary care physicians should be aware of asymptomatic patients with high pulse pressure and MNSI scores. Our findings also suggested that the importance of blood pressure measurement and foot examinations for early screening of PAD in the primary care setting.

\section{ACKNOWLEDGMENTS}

This study was supported in part by the grants from the National Science Council, Taiwan (NSC-98-2314-B-255004-MY3). We thank the Public Health Bureau of Chiayi, Taiwan for their support and help.

\section{REFERENCES}

1. American Diabetes Association. Peripheral arterial disease in people with diabetes. Diabetes Care 2003;26:3333-41.

2. McDermott MM, Mehta S, Greenland P. Exertional leg symptoms other than intermittent claudication are common in peripheral arterial disease. Arch Intern Med 1999;159:387-92.

3. Barretto S, Ballman KV, Rooke TW, Kullo IJ. Early-onset peripheral arterial occlusive disease: Clinical features and determinants of disease severity and location. Vasc Med 2003;8:95-100.

4. Adler AI, Boyko EJ, Ahroni JH, Smith DG. Lower-extremity amputation in diabetes. The independent effects of peripheral vascular disease, sensory neuropathy, and foot ulcers. Diabetes Care 1999;22:1029-35.

5. Jude EB, Oyibo SO, Chalmers N, Boulton AJ. Peripheral arterial disease in diabetic and nondiabetic patients: A comparison of severity and outcome. Diabetes Care 2001;24:1433-7.

6. Mohler ER $3^{\text {rd }}$, Treat-Jacobson D, Reilly MP, Cunningham KE, Miani M, Criqui MH, et al. Utility and barriers to performance of the ankle-brachial index in primary care practice. Vasc Med 2004;9:253-60.

7. Fowkes FG, Housley E, Macintyre CC, Prescott RJ, Ruckley CV. Variability of ankle and brachial systolic pressures in the measurement of atherosclerotic peripheral arterial disease. J Epidemiol Community Health 1988;42:128-33.

8. van Langen $H$, van Gurp J, Rubbens L. Interobserver variability of ankle-brachial index measurements at rest and post exercise in patients with intermittent claudication. Vasc Med 2009;14:221-6.

9. Rooke TW, Hirsch AT, Misra S, Sidawy AN, Beckman JA, Findeiss LK, et al. 2011 ACCF/AHA focused update of the guideline for the management of patients with peripheral artery disease (Updating the 2005 Guideline): A report of the American College of Cardiology Foundation/American Heart Association Task Force on practice guidelines. Circulation 2011;124:2020-45.

10. Eason SL, Petersen NJ, Suarez-Almazor M, Davis B, Collins TC. Diabetes mellitus, smoking, and the risk for asymptomatic peripheral arterial disease: Whom should we screen? J Am Board Fam Pract 2005; 18:355-61.

11. Aronow WS, Ahmed MI, Ekundayo OJ, Allman RM, Ahmed A. A propensity-matched study of the association of peripheral arterial disease with cardiovascular outcomes in community-dwelling older adults. Am J Cardiol 2009;103:130-5.

12. Khedara A, Kawai Y, Kayashita J, Kato N. Feeding rats the nitric oxide synthase inhibitor, L-N (omega) nitroarginine, elevates serum triglyceride and cholesterol and lowers hepatic fatty acid oxidation. J Nutr 1996;126:2563-7.

13. Moghtaderi A, Bakhshipour A, Rashidi H. Validation of Michigan neuropathy screening instrument for diabetic peripheral neuropathy. Clin Neurol Neurosurg 2006;108:477-81.

14. Fowkes FG, Murray GD, Butcher I, Heald CL, Lee RJ, Chambless LE, et al. Ankle brachial index combined with Framingham Risk Score to predict cardiovascular events and mortality: A meta-analysis. JAMA 2008;300:197-208.

15. Lange S, Diehm C, Darius H, Haberl R, Allenberg JR, Pittrow D, et al. High prevalence of peripheral arterial disease but low antiplatelet treatment rates in elderly primary care patients with diabetes. Diabetes Care 2003;26:3357-8. 
16. Rabia K, Khoo EM. Prevalence of peripheral arterial disease in patients with diabetes mellitus in a primary care setting. Med J Malaysia 2007;62:130-3.

17. Maeda Y, Inoguchi T, Tsubouchi H, Sawada F, Sasaki S, Fujii M, et al. High prevalence of peripheral arterial disease diagnosed by low ankle-brachial index in Japanese patients with diabetes: The Kyushu prevention study for atherosclerosis. Diabetes Res Clin Pract 2008;82:378-82.

18. Rhee SY, Guan H, Liu ZM, Cheng SW, Waspadji S, Palmes P, et al. Multi-country study on the prevalence and clinical features of peripheral arterial disease in Asian type 2 diabetes patients at high risk of atherosclerosis. Diabetes Res Clin Pract 2007;76:82-92.

19. Tseng $\mathrm{CH}$. Prevalence and risk factors of peripheral arterial obstructive disease in Taiwanese type 2 diabetic patients. Angiology 2003;54:331-8.

20. Hirsch AT, Hartman L, Town RJ, Virnig BA. National health care costs of peripheral arterial disease in the Medicare population. Vasc Med 2008;13:209-15.

21. Franklin SS. Cardiovascular risks related to increased diastolic, systolic and pulse pressure. An epidemiologist's point of view. Pathol Biol (Paris) 1999;47:594-603.

22. Knudsen ST, Poulsen PL, Hansen KW, Ebbehoj E, Bek T, Mogensen CE. Pulse pressure and diurnal blood pressure variation: Association with micro- and macrovascular complications in type 2 diabetes. Am J Hypertens 2002;15:244-50.

23. Zoppini G, Verlato G, Zamboni C, Venturi C, Gennaro N, Biasi V, et al. Pulse pressure and mortality from cerebrovascular diseases in type 2 diabetic patients: The Verona Diabetes Study. Cerebrovasc Dis 2007;23:20-6.

24. Zoppini G, Verlato G, Targher G, Bonora E, Trombetta M, Muggeo M. Variability of body weight, pulse pressure and glycaemia strongly predict total mortality in elderly type 2 diabetic patients. The verona diabetes study. Diabetes Metab Res Rev 2008;24:624-8.

25. Schram MT, Kostense PJ, Van Dijk RA, Dekker JM, Nijpels G,
Bouter LM, et al. Diabetes, pulse pressure and cardiovascular mortality: The hoorn study. J Hypertens 2002;20:1743-51.

26. Monami M, Vivarelli M, Desideri CM, Colombi C, Marchionni N, Mannucci E. Pulse pressure and prediction of incident foot ulcers in type 2 diabetes. Diabetes Care 2009;32:897-9.

27. Tseng CH. Pulse pressure as a risk factor for peripheral vascular disease in type 2 diabetic patients. Clin Exp Hypertens 2003;25:475-85.

28. Halon DA, Dobrecky-Mery I, Gaspar T, Azencot M, Yaniv N, Peled $\mathrm{N}$, et al. Pulse pressure and coronary atherosclerosis in asymptomatic type 2 diabetes mellitus: A 64 channel cardiac computed tomography analysis. Int J Cardiol 2010;143:63-71.

29. Bax G, Fagherazzi C, Piarulli F, Nicolucci A, Fedele D. Reproducibility of michigan neuropathy screening instrument (MNSI). A comparison with tests using the vibratory and thermal perception thresholds. Diabetes Care 1996;19:904-5.

30. Meves SH, Diehm C, Berger K, Pittrow D, Trampisch HJ, Burghaus I, et al. Peripheral arterial disease as an independent predictor for excess stroke morbidity and mortality in primary-care patients: 5-year results of the getABI study. Cerebrovasc Dis 2010;29:546-54.

31. Sen S, Lynch DR Jr., Kaltsas E, Simmons J, Tan WA, Kim J, et al. Association of asymptomatic peripheral arterial disease with vascular events in patients with stroke or transient ischemic attack. Stroke 2009;40:3472-7.

32. Topakian R, Nanz S, Rohrbacher B, Koppensteiner R, Aichner FT. High prevalence of peripheral arterial disease in patients with acute ischaemic stroke. Cerebrovasc Dis 2010;29:248-54.

33. Tseng $\mathrm{CH}$. An overview on peripheral vascular disease in blackfoot disease-hyperendemic villages in Taiwan. Angiology 2002;53:529-37.

Biomed J Vol. 36 No. 6

November - December 2013 